\title{
Pyruvate and glucose uptake by mouse ova and preimplantation embryos
}

\author{
H. J. Leese and Alison M. Barton \\ Department of Biology, University of York, York YO1 SDD, U.K.
}

\begin{abstract}
Summary. A method has been devised to measure the uptake of nutrients by small numbers of mouse ova and preimplantation embryos. The $1-5$ embryos were incubated as a group at $37^{\circ} \mathrm{C}$ in a small volume (at least $20 \mathrm{nl} / \mathrm{embryo}$ ) of culture medium on siliconized microscope slides under mineral oil. Serial $4 \mathrm{nl}$ samples were taken at 30 min intervals for $3 \mathrm{~h}$ and assayed for their content of glucose and pyruvate using an ultramicrofluorometric technique. Pyruvate uptake exceeded that of glucose in unfertilized and fertilized ova and in the developmental stages up to the blastocyst, when glucose became the predominant substrate. A component of the uptake of pyruvate by unfertilized ova was probably mediated by a carrier.
\end{abstract}

\section{Introduction}

Pyruvic acid is required to support the first and second cleavage divisions of the mouse embryo in culture (Biggers, Whittingham \& Donahue, 1967). Glucose is unable to support development until the 4-cell stage (Whitten, 1956, 1957; Brinster, 1965; Brinster \& Thomson, 1966). The reason for this early dependence on pyruvate is unknown, as is the nature of the switch in metabolism which later enables glucose to act as an energy source. Most of the studies into this problem have measured the uptake of radioactively-labelled substrates by large numbers of embryos $(50-250)$ to provide sufficient material for analysis (for review, see Brinster, 1973). The use of radioisotopes in metabolic experiments can introduce technical and interpretative problems, while averaging the data from large numbers of embryos may blur the differences between developmental stages. As a first approach to overcoming these difficulties, we have measured the uptake of pyruvate and glucose by small numbers (1-5) of mouse ova and preimplantation embryos using an ultramicrofluorescence technique originally devised to measure the adenine nucleotide content of single mouse embryos (Leese, Biggers, Mroz \& Lechene, 1984).

\section{Materials and Methods}

Unfertilized and fertilized ova, and embryos from the 2-cell stage to the blastocyst were flushed from the oviduct or uterus of randomly bred superovulated mice (strain LACA) with Medium M2 (Quinn, Barros \& Whittingham, 1982). This medium is buffered by Hepes and contains $5.56 \mathrm{~mm}$ glucose, $0.33 \mathrm{~mm}$-pyruvate and $23.3 \mathrm{~mm}$-lactate in addition to mineral salts and $4 \mathrm{mg}$ bovine serum albumin $/ \mathrm{ml}$. In the experiments on the various developmental stages the glucose concentration in the medium was reduced to $1 \mathrm{~mm}$. The 1 -cell stages were treated with $1 \mathrm{mg}$ hyaluronidase $/ \mathrm{ml}$ (Sigma London Chemical Company, Poole, Dorset, U.K.) to disperse the cumulus mass, and twice rinsed in fresh Medium M2. The 1-5 embryos were then placed as a group in a small volume of 
Medium M2 ( $\sim 20 \mathrm{nl} /$ embryo as judged by eye) on a siliconized microscope slide under a layer of mineral oil (Sigma). The slides were siliconized by dipping them for $30 \mathrm{sec}$ in $2 \%(\mathrm{v} / \mathrm{v})$ dimethyldichlorosilane (BDH, Poole, Dorset, U.K.), and the mineral oil was equilibrated overnight with $0.9 \%(\mathrm{w} / \mathrm{v}) \mathrm{NaCl}$ before use. The slides containing the embryo droplets were placed on a water-jacketed Perspex plate at $37^{\circ} \mathrm{C}$ and incubated in the dark for up to $3 \mathrm{~h}$. Samples of the incubation medium ( $380 \mathrm{pl}-4 \mathrm{nl}$ depending on the metabolite concentration) were removed at 30 -min intervals with calibrated micropipettes and analysed for glucose or pyruvate. The technique for constructing, calibrating and using micropipettes has been described by Mroz \& Lechene (1980). If the first 30-min sample was assayed for glucose, the second was assayed for pyruvate and so on, so that the uptake of both nutrients was measured in the same experiment. At the end of the experiments, the volume of the incubation medium remaining on the slide was measured by taking it up with a series of calibrated micropipettes.

The analytical method is a miniaturized version of the conventional enzymic methods of analysis, in which NADH or NADPH are generated or consumed in coupled reactions (Lowry \& Passoneau, 1972; Bergmeyer \& Gawehn, 1974). Instead of being carried out in cuvettes, the reactions are done in $25-30 \mathrm{nl}$ droplets on siliconized microscope slides under saline-saturated mineral oil, and the reaction products quantified with a fluorescent microscope with photomultiplier and photometer attachments (Leese, Biggers, Mroz \& Lechene, 1984).

The reagents had the following composition.

(1) Glucose assay: $3.7 \mathrm{mM}^{-\mathrm{MgSO}_{4}} .7 \mathrm{H}_{2} \mathrm{O}, 0.6 \mathrm{~mm}-\mathrm{NADP}, 0.5 \mathrm{~mm}$-ATP, $0.5 \mathrm{~mm}$-dithiothreitol, $12 \mathrm{U}$ hexokinase/ml (EC 2.7.1.1), $6 \mathrm{U}$ glucose 6-phosphate dehydrogenase/ml (EC 1.1.1.49), in 50 mM-EPPS buffer, (4-[2-Hydroxyethyl]-1-piperazine propane-sulfonic acid) $\mathrm{pH} 8.0$.

(2) Pyruvate assay: 0.075 mM-NADH, $28 \mathrm{U}$ lactate dehydrogenase $/ \mathrm{ml}$ (EC 1.1.1.27), in $50 \mathrm{~mm}$ EPPS buffer, $\mathrm{pH} 8 \cdot 0$.

Enzymes and coenzymes were obtained from Boehringer Mannheim, Lewes, East Sussex, U.K. $\alpha$ Cyano-4-hydroxycinnamate was obtained from the Aldrich Chemical Company, Milwaukee, Wisconsin, U.S.A. The final enzyme protein concentration was approximately 10 times greater than that used in conventional, macro-scale assays to compensate for the denaturation which is thought to occur at the oil-water interface of small droplets (Leese et al., 1984). All the reactions were started by the addition of the sample of incubation medium. The fluorescent microscope was a Vickers M17070 (Vickers Instruments, Haxby Road, York, U.K.) with 50-W mercury lamp, ultraviolet excitation filter and photomultiplier and digital microphotometer attachments. Most of the measurements were made with a $\times 10$ objective and Range 4 setting on the microphotometer. With these settings, the microscope measures the fluorescence of a circle of light $72 \mu \mathrm{m}$ in diameter, or approximately one fifth the diameter of a $20 \mathrm{nl}$ droplet.

It was found that prolonged exposure of the droplets to the light from the mercury lamp led to the oxidation of NADH and NADPH and a downward drift in the readings. The samples were therefore exposed for the minimum time required to obtain a stable reading $(\sim 3 \mathrm{sec})$, in which period the photo-oxidation was negligible. The glucose and pyruvate reactions went to completion in under $5 \mathrm{~min}$. The final readings were stable for at least $30 \mathrm{~min}$. There was a linear relationship between fluorescence and substrate concentration in the range $0-1 \mathrm{mM}$.

Experiments were carried out at the macro and ultramicro levels to determine whether there was any equilibration between the pyruvate and glucose in aqueous droplets and the overlying mineral oil, and whether it was necessary to saturate the oil with saline to prevent the droplets from evaporating. It was concluded that there was no significant loss of pyruvate and glucose (nor of ATP, lactate or glycogen) from such samples into the oil, and that the use of saline-saturated oil, while probably not essential with droplets in the range $20-30 \mathrm{nl}$, was nevertheless to be preferred.

The decrease in medium concentration of pyruvate and glucose was linear, and the rate of substrate uptake in $\mathrm{pmol} / \mathrm{embryo} / \mathrm{h}$ was calculated by regression analysis. A droplet of Medium M2 not containing embryos was always included in each experiment as a control for possible pyruvate or glucose losses, and any changes were subtracted from the embryo uptake values. 


\section{Results}

Pyruvate was readily taken up by unfertilized and fertilized ova at rates of $0 \cdot 86 \pm 0 \cdot 7(n=6)$ and $0.76 \pm 0.12(n=6) \mathrm{pmol} / \mathrm{embryo} / \mathrm{h}$ respectively. To determine the minimum volume of incubation medium required to support optimal rates of nutrient uptake, a series of experiments were carried out with one-cell embryos in which the volume of medium per embryo was varied from approximately 3 to $30 \mathrm{nl}$. In the range $2.5-7.5 \mathrm{nl}$, the rate of pyruvate uptake was $0.60 \pm 0.1(n=9)$ $\mathrm{pmol} / \mathrm{embryo} / \mathrm{h}$. In the ranges $7 \cdot 5-12 \cdot 5,12.5-17.5$ and $17.5-22.5 \mathrm{nl}$, the average uptakes were 0.73 , 0.85 and $0.73 \mathrm{pmol} / \mathrm{embryo} / \mathrm{h}$ respectively, indicating that competition for substrate was not occurring at these concentrations, and that a volume of approximately $20 \mathrm{nl} / \mathrm{embryo}$ was more than adequate to support optimal rates of nutrient uptake.

Pyruvate uptake remained near to the 1-cell values in the 2-cell, 4-cell, 8-16-cell and morula stages of development before decreasing to $0.31 \pm 0.19(n=7)$ in the blastocyst (Text-fig. 1). Glucose uptake by unfertilized ova was barely detectable, and remained $<0.5 \mathrm{pmol} / \mathrm{embryo} / \mathrm{h}$ in the stages through to the morula before increasing dramatically to $3.4 \pm 0.54(n=9)$ in the blastocyst.

Unfertilized ova were used to investigate the dependence of pyruvate uptake on its concentration in the incubation medium (Text-fig. 2). While the rate of pyruvate uptake increased with increasing medium concentration, the relationship did not conform to that expected of either simple or facilitated diffusion, but rather to a combination of the two processes, with the one superimposed upon the other. Evidence for this came when the experiments were repeated in the presence of $1 \mathrm{~mm}-\alpha$-cyano-4-hydroxycinnamate, which inhibits the facilitated uptake of pyruvate by red blood cells, mitochondria and other systems (Halestrap \& Denton, 1974). In the presence of this inhibitor, pyruvate uptake was barely detectable at medium concentrations of 0.33 and 0.66 $\mathrm{mM}$ but was progressively taken up at higher concentrations in a manner suggestive of simple diffusion (lower trace in Text-fig. 2).

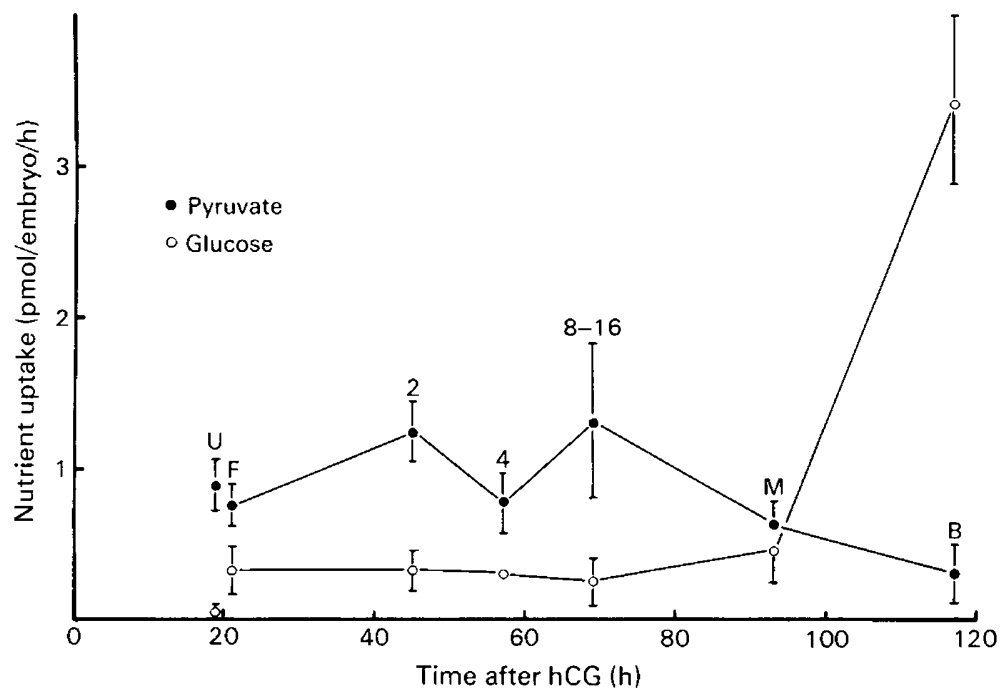

Text-fig. 1. The uptake of pyruvate and glucose by unfertilized (U) and fertilized (F) mouse ova, and the 2-cell (2), 4-cell (4), 8-16-cell (8-16), morula (M) and blastocyst (B) stages of development. Values are mean \pm s.e.m. of at least 6 determinations except for the 4-cell values which are the means of 3 (pyruvate) and 2 (glucose) determinations. 


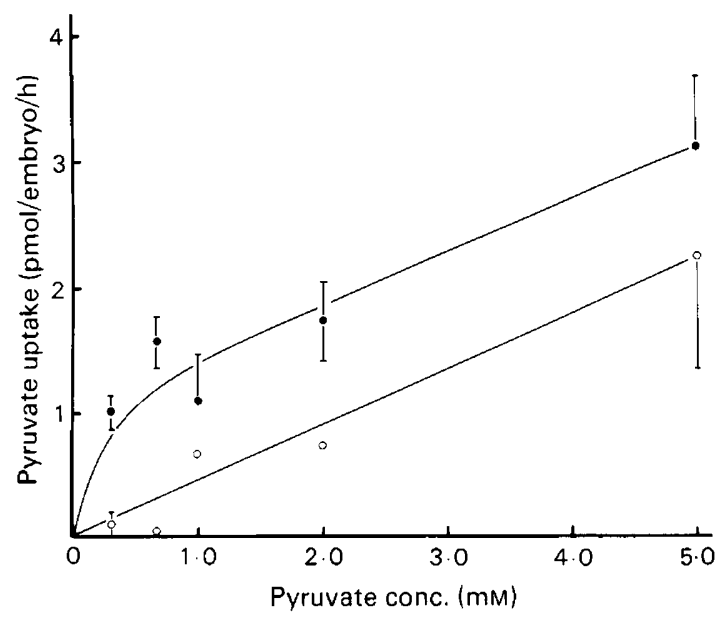

Text-fig. 2. The uptake of pyruvate by unfertilized mouse ova as a function of medium pyruvate concentration in the presence $(O)$ and absence (O) of 1 mM- $\alpha$-cyano-4hydroxycinnamate. Control values are mean \pm s.e.m. of at least 6 determinations. Inhibitor values are mean of 3 determinations \pm s.e.m. or 2 determinations.

\section{Discussion}

We have shown that the uptake of two nutrients essential for early mammalian development may readily be detected using only 1-5 embryos. The pattern of the results is in agreement with those of others in showing that pyruvate is the preferred substrate in the early preimplantation stages, with glucose predominating in the blastocyst. Whereas pyruvate is likely to be used as an energy source by preimplantation embryos, Pike \& Wales (1982a, b) have shown that much of the glucose is incorporated into glycogen. The nature of the block in glycolysis which prevents glucose from acting as a sufficient energy source for early embryos has been examined in some detail by Barbehenn, Wales \& Lowry $(1974,1978)$. The block may reside at the level of glucose transport, hexokinase or phosphofructokinase, or involve some combination of these sites. The ratio of ATP to ADP, which falls sharply with development, may also be important (Quinn \& Wales, 1973; Leese et al., 1984).

Although direct comparisons are difficult due to the use of different methods, the uptake values are slightly lower than those summarized by Brinster (1973), but compare well with those of Nilsson, Östensson, Eide \& Hellerström (1980) for glucose utilization by mouse blastocysts (4·1 $\mathrm{pmol} / \mathrm{embryo} / \mathrm{h}$ at a medium concentration of $16.7 \mathrm{~mm}$ compared with $3.4 \mathrm{pmol} / \mathrm{embryo} / \mathrm{h}$ at a concentration of $1 \mathrm{~mm}$ in the present work). Renard, Philippon \& Menezo (1980) found much larger glucose uptakes in bovine blastocysts obtained from cows 10-11 days after oestrus (5.8 $\mu \mathrm{g} / \mathrm{embryo} / \mathrm{h}$ ) reflecting the greater size of embryos at this age. Significantly, more bovine blastocysts which showed a glucose uptake developed in vivo after cervical transfer than did those with no glucose uptake, suggesting that this might provide the basis of a non-invasive technique for assessing embryo viability.

The increase in glucose uptake was delayed until the blastocyst stage (Text-fig. 1) whereas others have indicated that the transition to a glucose-based metabolism is apparent in the morula (Wales, 1975). Part of the explanation for these discrepencies could lie in the use of mixtures of substrates (i.e. glucose, pyruvate and lactate) in the present experiments, and the use of single substrates in some of the earlier studies. A further possibility, which it is hoped to investigate, is the contribution of individual embryo differences to the variation in the results. This will be done by 
culturing single or small numbers of embryos and measuring their pyruvate and glucose uptakes throughout the various developmental stages, returning them between measurement to the culture medium. Barbehenn et al. (1978) have commented on the desirability of assessing such individual differences.

Pyruvate uptake as a function of medium pyruvate concentration and the results with $\alpha$-cyano4-hydroxycinnamate, strongly suggested that a component of pyruvate transport into unfertilized ova was carrier-mediated. Indirect evidence in support of this was obtained by Wales \& Whittingham (1967), who found that pyruvate was accumulated faster than lactate by 1- and 2-cell mouse embryos.

We thank the Science and Engineering Research Council for a research grant.

\section{References}

Barbehenn, E.K., Wales, R.G. \& Lowry, O.H. (1974) The explanation for the blockade of glycolysis in early mouse embryos. Proc. natn. Acad. Sci. U.S.A. 71, 1056-1060.

Barbehenn, E.K., Wales, R.G. \& Lowry, O.H. (1978) Measurement of metabolites in single preimplantation embryos; a new means to study metabolic control in early embryos. J. Embryol. exp. Morph. 43, $29-46$.

Bergmeyer, H.U. \& Gawehn, K. (1974) Methods of Enzymatic Analysis, 2nd English edn. Academic Press, New York.

Biggers, J.D., Whittingham, D.G. \& Donahue, R.P. (1976) The pattern of energy metabolism in the mouse oocyte and zygote. Proc. natn. Acad. Sci. U.S.A. 58, 560-567.

Brinster, R.L. (1965) Studies on the development of mouse embryos in vitro. II. The effect of energy source. J. exp. Zool. 158, 59-68.

Brinster, R.L. (1973) Nutrition and metabolism of the ovum, zygote and blastocyst. In Handbook of Physiology, Section 7, Vol. II, Part 2, pp. 165-185. Eds R. O. Greep \& E. B. Astwood. Am. Physiol. Soc., Washington, D.C.

Brinster, R.L. \& Thomson, J.L. (1966) Development of eight-cell mouse embryos in vitro. Expl Cell Res. 42, 303-315.

Halestrap, A.P. \& Denton, R.M. (1974) Specific inhibition of pyruvate transport in rat liver mitochondria and human erythrocytes by $\alpha$-cyano-4-hydroxycinnamate. Biochem. J. 138, 313-316.

Leese, H.J., Biggers, J.D., Mroz, E.A. \& Lechene, C. (1984) Nucleotides in a single mammalian ovum or preimplantation embryo. Analyt. Biochem. (in press).

Lowry, O.H. \& Passoneau, J.V. (1972) A Flexible System of Enzymatic Analysis. Academic Press, New York.
Mroz, E.A. \& Lechene, C. (1980) Fluorescence analysis of picoliter samples. Analyt. Biochem. 102, 90-96.

Nilsson, B.O., Östensson, C.G., Eide, S. \& Hellerström, C. (1980) Utilization of glucose by the implanting mouse blastocyst activated by oestrogen. Endokrinologie 76, 82-93.

Pike, I.L. \& Wales, R.G. (1982a) Uptake and incorporation of glucose especially into the glycogen pools of preimplantation mouse embryos during culture in vitro. Aust. J. biol. Sci. 35, 195-206.

Pike, I.L. \& Wales, R.G. (1982b) Turnover of carbon pools labelled with $\left({ }^{14} \mathrm{C}\right)$ glucose during in vitro culture of preimplantation mouse embryos. Aust. $J$. biol. Sci. 35, 637-644.

Quinn, P. \& Wales, R.G. (1973) The effect of culture in vitro on the levels of adenosine triphosphate in preimplantation mouse embryos. J. Reprod. Fert. 32, 231-241.

Quinn, P., Barros, C. \& Whittingham, D.G. (1982) Preservation of hamster oocytes to assay the fertilizing capacity of human spermatozoa. J. Reprod. Fert. 66, 161-168.

Renard, J.-P., Philippon, A. \& Menezo, Y. (1980) In-vitro uptake of glucose by bovine blastocysts. J. Reprod. Fert. 58, 161-164.

Wales, R.G. (1975) Maturation of the mammalian embryo: biochemical aspects. Biol. Reprod. 12, 6681.

Wales, R.G. \& Whittingham, D.G. (1967) A comparison of the uptake and utilization of lactate and pyruvate by one- and two-cell mouse embryos. Biochim. Biophys. Acta 148, 703-712.

Whitten, W.K. (1956) Culture of tubal mouse ova. Nature, Lond. 177, 96-97.

Whitten, W.K. (1957) Culture of tubal ova. Nature, Lond. 179, 1081-1082. 